\title{
THE ODF APPROXIMATION FROM POLE FIGURES WITH THE AID OF THE ADC METHOD
}

\author{
K. Pawlik*, J. Pospiech*, K. Lücke** \\ * Instytut Podstaw Metalurgii PAN, Kraków, Poland \\ * Institut für Metallkunde und Metallphysik, RWTH Aachen, FRG
}

\section{ABSTRACT}

The ADC method reported earlier by the present authors is one of the direct methods for ODF approximation from pole figures. It provides very good approximations of the ODF for typical rolling and recrystallization textures, but, as demonstrated for the very ghost sensitive model of Matthies, it leads to weak ghost errors for weak textures containing a large fraction of the constant component. Here, an iteration procedure for the ADC method essentially differing from the previous one will be presented which yields ghost-free ODFs for a wide range of possible textures.

\section{THE ADC METHOD}

The basic soncepts of the direct approximation of the ODF from pole figures - i.e. not using series expansion - were developed a long time ago by Williams (1968) $)^{1}$, Ruer and Baro $(1977)^{2}$ and, above all, by Imhof (1978) ${ }^{3}$. Improved versions of direct methods have been proposed more recently: the WIMV method by Matthies and Vinel $(1982)^{4}$ and the ADC method by Pawlik, Pospiech and Lücke (1987) $)^{5,6,7}$. These methods are based on the discrete form of the fundamental equation. The problem of the ODF approximation is there considered directly in the orientation space making use of the fact that the discrete hemisphere of the pole figure can be represented in the ODF space by families of projection tubes. Through these projection tubes information from pole figures is transfered into the orientation space by applying a process of iteration which will result in an approximation of a discrete ODF.

A solution of the ODF calculation problem is found when the calculated ODF yields the original (e.g. the experimental) pole figures. As it is known there exists a basic ambiguity in the sense that a whole range of solutions is able to satisfy this condition. The iteration procedure always leads to a solution, but, in general, this solution deviates from the desired true ODF by showing effects of ghost adulterations. For this reason additional information or conditions need to be introduced into the iteration procedure to control the way of ODF approximation. This has been discussed and demonstrated by Matthies ${ }^{8}$ by applying the WIMV method to appropriately chosen model ODFs.

When developing the ADC method attention has been given to an exact as possible utilization of all essential relations between the discrete hemisphere of the pole figures and the discrete ODF space. Consequently projection tubes have been applied in the ADC method instead of projection lines which were adopted in the method by Williams and also in the WIMV method in order to simplify the calculations. In contrast to the vector method and the method by Imhof ${ }^{9}$ the division of the ODF space into discrete cells in the ADC method does not depend on the division of the pole figure sphere (hence the name 
ADC - Arbitrary Defined Cells). The cross section of a tube is fitted in the ADC method only to the size and shape of the corresponding domain of the discrete division of the hemisphere. This geometry of the tubes in the discrete ODF space served as basis for the derivation of the iteration operator which turns out to differ from that introduced by Imhof only through a geometrical factor. In the iteration process the non-negativity of the ODF (and thereby also the zero-fields in the pole figures) are automatically taken into account. Also the well-founded assumption that random pole figures indicate a random ODF which stabilizes the iteration process is satisfied.

The previous version of the ADC method worked without further additional information and the appearance of ghost errors for weak textures implies that the conditions controlling the iteration process were not suited to sufficiently suppress these errors in the whole range of possible textures. The removal of this difficulty requires the introduction of additional assumptions. Such a well founded modification of the ADC method will now be presented. There the iteration process is composed of three stages. The transition from one stage to the next is determined by the value of the mean relativ error ${ }^{7} R^{(n)}$ or the value $\Delta R P^{(n)} / R P^{(n)}$ which both represent convergence measures after the $n$-th iteration step.

\section{STAGE I ITERATION}

The aim of this stage is to adjust the several experimental pole figures and to average the statistical error as well as to derive a first approximation of the ODF.

The operator of the stage $I$ is identical to that which have been described in the foregoing version of the ADC method:

$$
\begin{gathered}
I_{a}^{(1)}=<\left[\sum_{T_{\text {twoc }}} U_{\text {akie }} P_{\text {kie }}^{\exp }\right]^{\lambda}>_{e=1 \ldots E_{1}}>_{i=1 \ldots I} \\
I_{a}^{n+1)}=I_{I} f_{a}^{(n)} \ll\left[\sum_{T_{\text {uac }}} U_{\text {akie }} \frac{P_{\text {kie }}^{\exp }}{P_{\text {kie }}^{(n)}}\right]>_{e=1 \ldots E_{i}}>_{i=1 \ldots I}
\end{gathered}
$$

Here means $P_{k i}$ the value of the kie domain of the pole figures (i numerates the pole figure, $k$ the domain in this pole figure and e numerates symmetrically equivalent poles), $f_{a}$ the value of the ODF assigned to the cell $C_{a}$, and $T_{k i e} / C_{a}$ indicates that the summation proceeds along those tubes $T_{\text {bi }}$ which intersect the cell $C_{a} . V_{a}$ is the volume of the cell $C_{a}, V_{a j j e}$ the volume of the part of the cell intersected by the tube and $V_{\text {alco }} / V_{2}$ the volume fraction of the intersected part. $\lambda$ is a parameter which influences the rate of convergence of the iteration process. It is chosen by experience and is mostly set to be $\approx 2$.

The $P_{\text {bie }}$ values of the pole figures transferred by the tubes are assigned to the cells with taking into account the volume fraction of the part of the cell intersected by the tube. In the iteration procedure the geometrical averaging is used. In averaging each family ( $i=$ constant, $e=$ constant) of tubes is taken 
into account. After each iteration step the ODF is normalized.

\section{STAGE II ITERATION} iteration:

The pole figures adjusted in stage I serve as starting data for stage II of

$$
{ }_{n} P_{k i e}={ }_{I} P_{k i e}^{(n)}
$$

The iteration proceeds as follows. For each family of tubes (i,e) the value of the orientation density ${ }_{11} \mathrm{f}_{\mathrm{a}}^{(1)}$ in each cell $\mathrm{C}_{\mathrm{a}}$ is calculated, and always the smallest value is chosen from the set of values resulting for the various families of tubes intersecting the given cell a:

$$
{ }_{\| I} f_{a}^{(1)}=\operatorname{Min}\left[\sum_{T_{\text {Hedc }}} U_{\text {akie }}{ }_{\|} P_{k i e}\right] \rightarrow i=i_{m}, \quad e=e_{m}
$$

The so selected values $f_{a}$ are indicated by the indices $i=i_{m}, e=e_{m}$. After normalization in the ODF space pole figures are recalculated and next the value of the operator $Q_{I I}$ for $i=i_{m}$ and $e=e_{m}$

$$
Q_{I I}^{(n)}=\left[\sum_{T_{\text {kidc }}} U_{\text {akie }} \frac{{ }_{I I} P_{k i e}}{{ }_{I I} P_{k i e}^{(n)}}\right]^{\lambda}<_{i=i_{m}, e=e_{m}}
$$

is determined. Now depending on the value of $Q_{\text {II }}$ the densities in the cells for the next iteration are selected as follows

$$
{ }_{I I} f_{a}^{(n+1)}=\left\{\begin{array}{l}
f_{a}^{(n)} \text { if } Q_{I I}^{(n)} \geq 1 \\
{ }_{\| I} f_{a}^{(n)} Q_{I I}^{(n)} \text { if } Q_{I I}^{(n)}<1
\end{array}\right.
$$

In this procedure the values of $f_{a}$ larger than the true (e.g. experimental) values (i.e. $Q_{11}<1$ ) are diminished. After each iteration step the ODF is normalized then giving again the values for the next step of iteration. The aim of stage II is the approximation of the lower level ODF densities. It develops an idea presented earlier by Imhof ${ }^{f}$ but not utilized yet in iteration processes.

\section{STAGE III ITERATION}

After convergence of the iteration in stage II the pole figures from the second stage are transferred to the third stage

$$
{ }_{1 I I} P_{k i e}={ }_{n} P_{k i e}^{(n)}
$$

and the iteration proceeds in the following manner: 


$$
\begin{aligned}
& \text { III } P_{a}^{(1)}=\left\{\begin{array}{l}
f_{a}^{(n)} \text { for }{ }_{I I} f_{a}^{(n)} \geq 1 \\
{ }_{I I} f_{a}^{(n)} \text { for }{ }_{\text {II }} f_{a}<1
\end{array}\right. \\
& { }_{I I I} P_{a}^{(n+1)}=\left\{\begin{array}{llll}
I I I & f_{a}^{(n)} \cdot Q_{I I I}^{(n)} & \text { if }{ }_{\text {III }}^{(n)} f_{a}^{(n)} \geq 1 \\
I I I & f_{a}^{(n)} & \text { if } \text { III } f_{a}^{(n)}<1
\end{array}\right.
\end{aligned}
$$

where

$$
Q_{I I I}^{(n)}=<<\left[\sum_{T_{\text {tatc }}} U_{a k i e} \frac{\text { III } P_{k i e}}{I I I P_{k i e}^{(n)}}\right]^{\lambda}>_{e=1 \ldots E_{i}}>_{i=1 \ldots I}
$$

After each iteration step the ODF is normalized. The aim of stage III is to adjust the f-values of the various cells with respect to each other, which until here having been considered only individually. In the range of large orientation densities the values will be diminished if the calculated values are larger than the true ones $\left(Q_{I I I}<1\right)$ and increased if they are smaller $\left(Q_{I I I}>1\right)$, whereas no changes are made in the range of small densities. One sees that the iteration procedures in the stages II and III select from the range of possible solutions such ones in which the background (phon) is large and the number of low peaks small. As will be discussed more thoroughly in a subsequent paper, this is the above mentioned additional condition controlling the strategy of iteration. It is similar to the condition underlying WIMV.

\section{SOME TEST RESULTS}

The method has been tested by using model ODFs composed of Gausstype components around $\{100\}<001>,\{110\}<112>,\{213\}<364>$, $\{112\}<111\rangle$ and a constant component $\delta$ representing the backround. Their volume fractions were set to be $9 \%, 18 \%, 36 \%, 18 \%$ and $19 \%$, respectively and their widths $\psi$ (at half of the height of the maximum) to be equal for all these components. Taking into account the multiplicity of the components, in that case the height of their maxima would come out to be equal (except $\delta$ ). Three ODF models have been used with $\psi$ being either $10^{\circ}, 16^{\circ}$ or $20^{\circ}$. From each of these models 4 pole figures $\{100\},\{110\},\{111\},\{113\}$ were calculated. ODF test calculations were performed using $N=4,3$ or 2 of these pole figures, either complete ones $\left(\alpha_{\max }=90^{\circ}\right)$ or incomplete ones ranging up to $\alpha_{\max }=80^{\circ}$, $70^{\circ}$, or $60^{\circ}$.

The test results are characterized as follows:

(1) In the orientations distribution calculated for all $3 \times 4 \times 3=36$ cases no traces of ghost errors were observed.

(2) The quality of the reprocuded ODF depended neither on the number of the used pole figures nor on the cutting angle $\alpha_{\max }$ also controlling the investigated amount of input data. 
(3) For various $\mathrm{N}$ or $\alpha_{\max }$ irregular deviations of the heights of maxima (independent of $\mathrm{N}$ and $\alpha_{\max }$ ) are observed, but these deviations decrease with the increase of the width $\psi$. Deviations up to $10 \%$ are found for $\psi=10^{\circ}$, to $5 \%$ for $\psi=16^{\circ}$, to $4 \%$ for $\psi=20^{\circ}$.

Fig. 1 gives an example for the test results based on the model ODF for $\psi=16^{\circ}$. It shows that there are practically no visible differences between the model ODF and the calculated ODF.

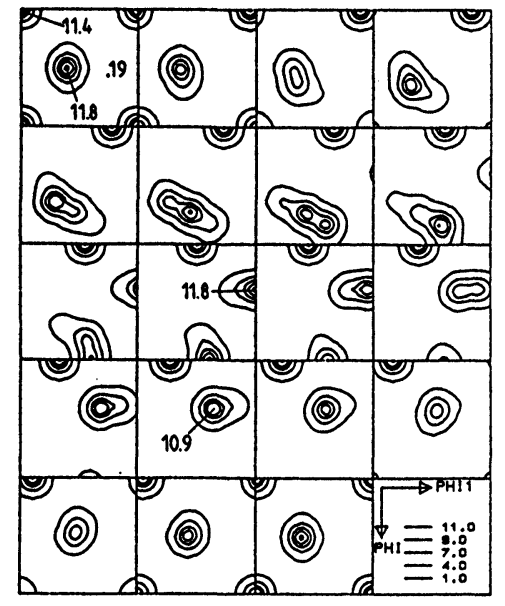

a)

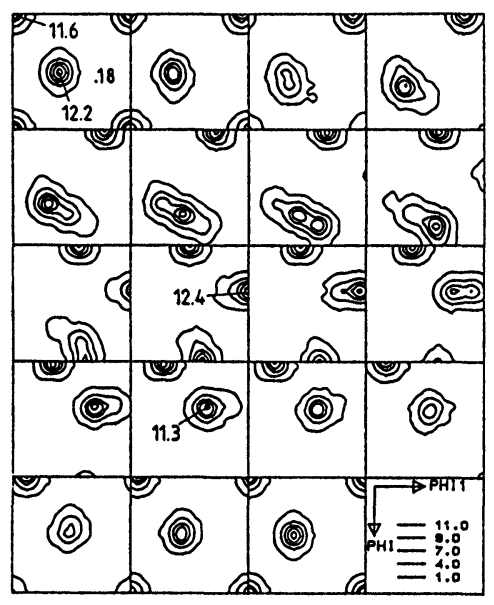

c)

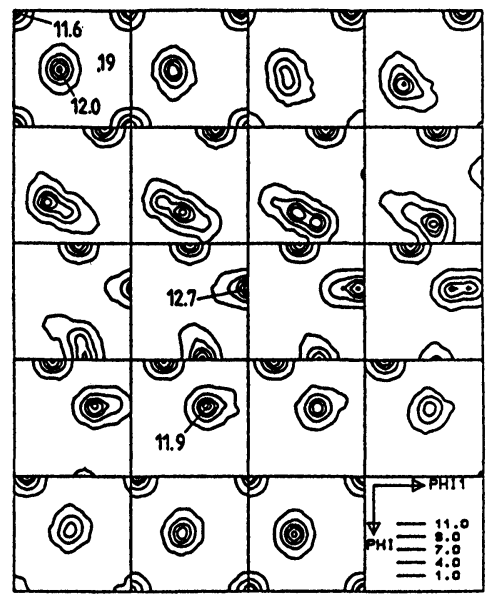

b)

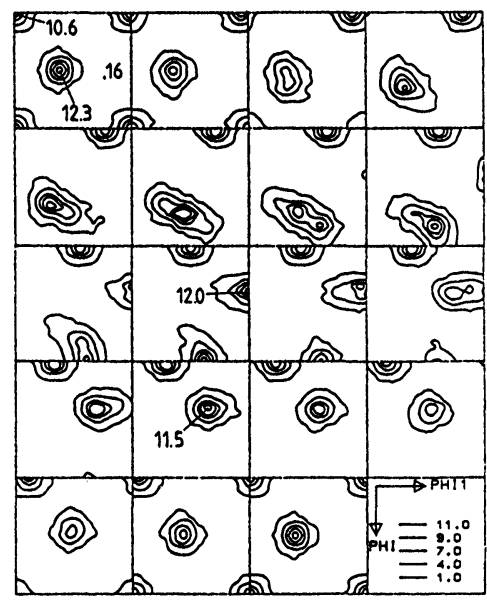

d)

Fig.1. Model $O D F$ (a) and ODF recalculations with the ADC method for 4 pole figures and different ranges $\alpha_{\max }$ : (b) $\alpha_{\max }=90^{\circ}$, (c) $\alpha_{\max }=80^{\circ}$, (d) $\alpha_{\max }=60^{\circ}$. 

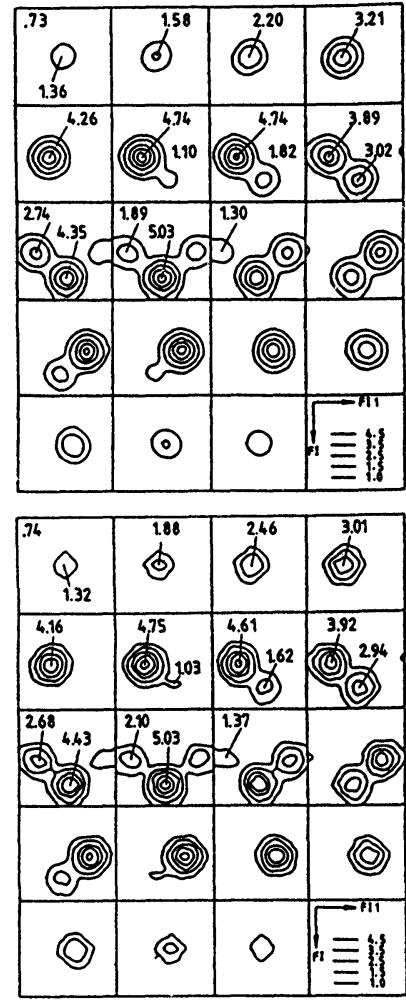

a)

C)

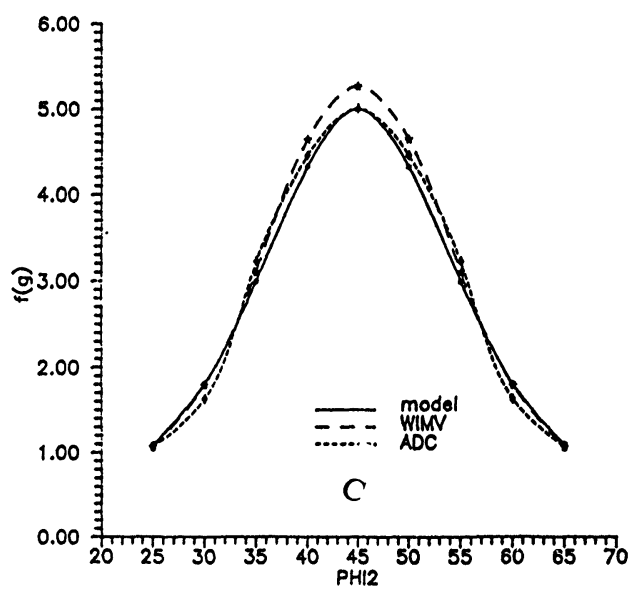

Fig.2. (a) Model ODF proposed by Matthies composed of a Gaussian component with $\psi=20^{\circ}$ in the Cube twin position $\{122\}<221>(27 \%)$ and of a constant component (73\%).

(b) The model function reproduced by the $A D C$ method.

(c) Comparison of $O D F$ values along a skeleton line.

Fig. 2 shows results of an other important test obtained for a model function used in the test calculation of the WIMV method by Matthies ${ }^{8}$. This model of a weak texture is made up of a Gaussian-type component with the maximum in the twin of the Cube position and a high background. Since the Cube position has a high multiplicity, it will lead to high density of a ghost in this position so that the reproduction of this model possesses a very high sensitivity with respect to the appearance of ghost errors. In the ODF reproduced by the ADC method (Fig.2b) the ghost could not be recognized which again demonstrates the high effectivity of this method. Fig.2c shows ODF values of the model ODF (Fig.2a) and obtained by the WIMV and by the ADC method (Fig.2b) along skeleton lines.

\section{REFERENCES}

[1] R.O. Williams, J. Appl. Phys. 39, 2024 (1968)

[2] D. Ruer, R. Baro, Adv. X-ray Analysis 20, 187 (1977)

[3] J. Appl. Cryst. 10, 458 (1977)

[4] S. Matthies, G.W. Vinell, phys. stat. sol. (b) 112, K111,K115 (1982)

[5] K. Pawlik, phys. stat. sol. (b) 134, 477 (1986)

[6] K. Pawlik, J. Pospiech, Proc. Conf. "Theoretical Methods in Texture Analysis", Clausthal-Zellerfeld, 1986, p.127

[7] K. Pawlik, J. Pospiech, K. Lücke, Proc. ICOTOM 8, Santa Fe, 1987, p. 105

[8] S. Matthies, Proc. ICOTOM 8, Santa Fe, 1987, p.37

[9] J. Imhof, Texture and Microstructure 6 ,73 (1982) 